Trauma Berufskrankh 2007 · 9:149-156

DOI 10.1007/s10039-006-1150-y

(c) Springer Medizin Verlag 2007

Erratum zu: Trauma Berufskrankh

$2007 \cdot 9$ [Suppl 2]:S237-\$244

In der Druckversion dieses Beitrags war durch einen technischen Fehler die Textfolge auf den Seiten S241/S242 vertauscht worden. Anbei finden Sie die korrekte Version.

Gleichzeitig stellen wir die Institutsangabe sowie die Adresse des korrespondierenden Autors richtig:

Chirurgische Klinik und Poliklinik I,

Universität Leipzig, Leipzig

\section{Hintergrund}

Epidemiologie, Ätiopathogenese. In Deutschland werden jährlich bis zu 8000 schwer wiegende Wirbelsäulenverletzungen im Bereich der thorakolumbalen Wirbelsäule beschrieben. Von diesen 70$80 \%$ aller relevanten Wirbelsäulenverletzungen sind mehr als die Hälfte in Höhe des sensiblen Übergangs von physiologischer Kyphose der Brustwirbelsäule zur Lendenlordose lokalisiert. Dabei ist der 1. Lendenwirbelkörper - in bis zu 50\% der Fälle - am häufigsten betroffen, gefolgt von den benachbarten Wirbelkörpern $[5,13,15]$. 90\% der Verletzungen sind mono- oder bisegmentale Wirbelsäulenverletzungen, seltener liegen Mehrsegment- oder Mehretagenverletzungen vor. Die häufigsten Erscheinungsformen sind mit 60-70\% die Kompressionsfrakturen (Typ A), gefolgt von Flexions-DistraktionsVerletzungen (Typ B) und den hochgradig instabilen Rotationsverletzungen (Typ C).

In der Mehrzahl sind in bis zu 70\% der Fälle Männer betroffen, wobei der Altersgipfel meist zwischen dem 20. und 40. Lebensjahr liegt. Die 3 häufigsten Unfallursachen sind

- Sturz aus der Höhe,

- Verkehrsunfälle und

- Sportunfälle $[5,15]$.

Im höheren Lebensalter führen häufig bereits banale Stürze aufgrund osteoporotisch reduzierter Tragfähigkeit zu Wirbelfrakturen.

\author{
S. Katscher $\cdot$ C. Josten \\ Chirurgische Klinik und Poliklinik III, Universität Leipzig, Leipzig
}

\title{
Wirbelsäulenverletzungen des thorakolumbalen Übergangs
}

\section{Versorgungsstrategien}

Änderung der Behandlungsstandards. Die Therapie von Verletzungen der Brustund Lendenwirbelsäule hat in der vergangenen Dekade eine permanente Entwicklung erfahren. Stabile Frakturen mit erhaltener Integrität des Spinalkanals werden ohne länger dauernde Immobilisation zunehmend frühfunktionell behandelt.

Bei instabilen Verletzungen besteht über die Notwendigkeit einer operativen Behandlung zur Wiederherstellung des physiologischen Alignements sowie zur Vermeidung von Spätschäden weit gehende Einigkeit. Allerdings sind die allei- nige Reposition und dorsale Instrumentierung längst nicht mehr der „Goldstandard“. Ursächlich dafür ist der in zahlreichen Studien $[9,13,15,19]$ beobachtete sekundäre Korrekturverlust in den der Fraktur benachbarten Bandscheibenregionen. Somit wurde den im Rahmen des Traumas durch Einsprengung von Wirbelkörperfragmenten bzw. Einblutung mitverletzten Bandscheiben mehr Beachtung geschenkt. Die dieses Problem adressierende und zwischenzeitlich häufig durchgeführte Spongiosaplastik nach Daniaux [8] konnte die in sie gesetzten Erwartungen
Abb. 1 a Karbontisch mit typischen Lagerungsmaterialien für den ventralen Durchhang, $\mathbf{b}$ initiales Röntgenbild und $\mathbf{c}$ präoperatives Durchleuchtungsbild zeigen Effektivität der Lagerung mit bereits weitgehend wiederhergestelltem lokalem Alignement

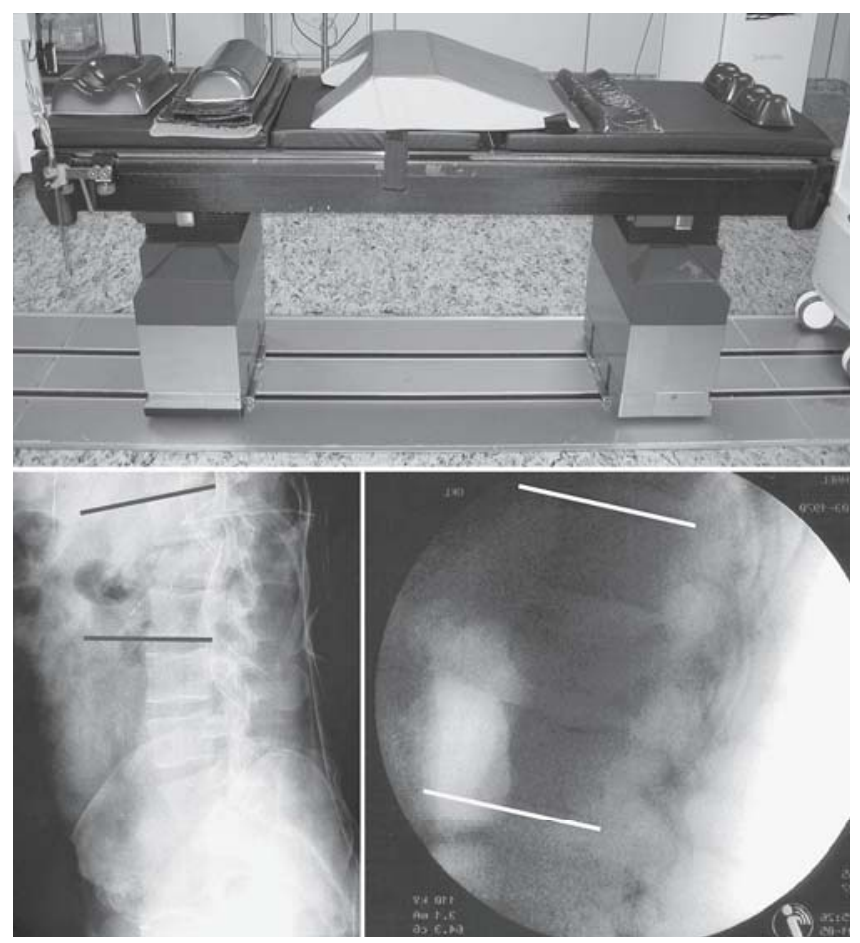




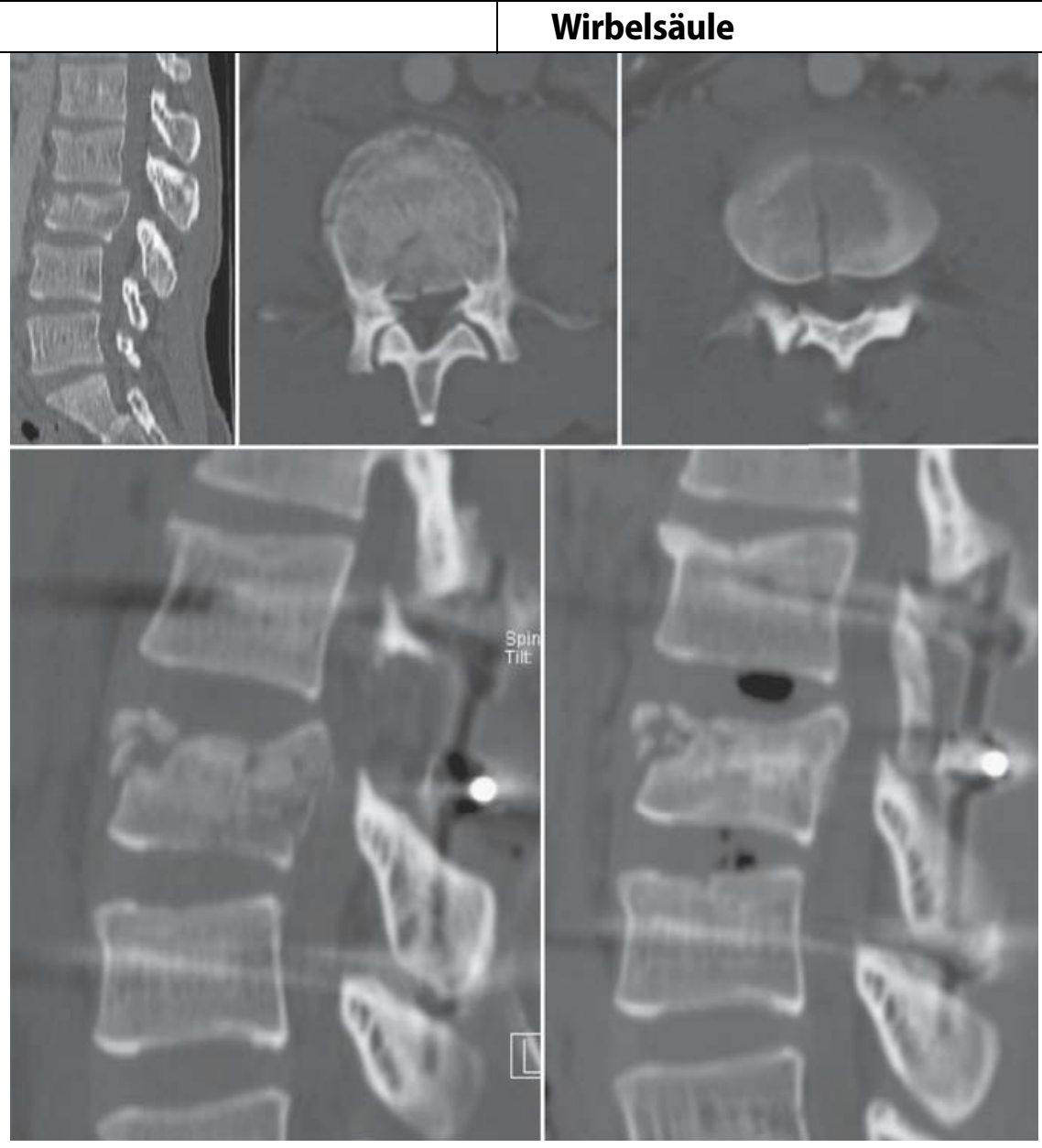

Abb. $2 \Delta$ a-c Typ-B-Verletzung mit Berstungsspaltbruch bei polytraumatisiertem 33-jährigem Patienten nach Sturz aus etwa $10 \mathrm{~m}$ Höhe, $\mathbf{d}$ direkt postoperatives $\mathrm{CT}$, keine sichtbare Veränderung im Bandscheibenraum, e 6 Wochen später angefertigtes Planungs-CT zur additiven ventralen Stabilisierung mit in beiden Bandscheibenräumen deutlichem Vakuumphänomen als Hinweis auf strukturelle Bandscheibenläsion (Anmerkung: Bei jüngeren Patienten mit guter Knochenqualität führen wir in Abhängigkeit vom Allgemeinzustand und Wunsch des Patienten die additive ventrale Stabilisierung entweder während des stationären Primäraufenthalts oder im Intervall nach etwa 6 Wochen durch)

nicht erfüllen. Stattdessen wurden zusätzlich zu konventionellen kortikospongiösen Knochenspänen mit steigender Resonanz expandierbare Cages, insbesondere zur biund mehrsegmentalen Defektüberbrückung mit oder ohne zusätzliche ventrale Instrumentation in den klinischen Gebrauch eingeführt. Gleichzeitig wurden die klassischen Operationszugänge, insbesondere ventral, mit dem Ziel eines geringeren Operationstraumas minimiert. Endoskopische oder endoskopisch assistierte Verfahren [6] zählen mittlerweile zum Standardrepertoire vieler Wirbelsäulenzentren. Zur Erhöhung der Sicherheit für Patient und Operateur wurden kostenintensive Navigationssysteme entwickelt, welche in einigen Kliniken ebenfalls fest im individuellen therapeutischen Regime etabliert sind.
Auch die Therapie osteoporotischer Frakturen beim älteren Menschen hat sich v. a. durch die Einführung der schmerztherapeutisch hocheffizienten Kyphoplastie $[2,3,14]$ deutlich in Richtung einer operativen Versorgung gewandelt.

\section{Diagnostik und Klassifikation}

\section{Maßnahmen am Unfallort}

Diagnostik und Therapie jeglicher Wirbelsäulenverletzung beginnen bereits am Unfallort, und speziell für den unkooperativen, bewusstlosen oder polytraumatisierten Patienten gilt, dass so lange von einer Wirbelsäulenverletzung auszugehen ist, bis in der Klinik durch die radiologischen Untersuchungen das Gegenteil bewiesen werden kann. Bei entspre- chendem Unfallmechanismus und klinischen Zeichen wie Rückenschmerzen, Prellmarken oder gar neurologischen Ausfällen ist somit vom Rettungsdienst auf eine achsgerechte Bergung und Lagerung des Patienten zum Transport auf der Vakuummatratze zu achten. Sollten eine Intubation und Beatmung des Patienten vor Ort erforderlich werden, sind vorher vom Notarzt eine orientierende neurologische Untersuchung und entsprechende Dokumentation vorzunehmen.

\section{Maßnahmen in der Klinik}

Beim polytraumatisierten, analgosedierten und beatmeten Patienten kann die neurologische Untersuchung auf ein Minimum reduziert werden. Ansonsten sind die exakte klinische und neurologische Reevaluierung inklusive Prüfung des Analsphinktertonus obligat [23]. Da Wirbelsäulenverletzungen mit begleitenden neurologischen Ausfällen in der Regel Ausdruck eines hochenergetischen Traumas sind, liegen auch bei scheinbarem Monotrauma häufig initial okkulte thorakale oder abdominelle Begleitverletzungen vor. Dementsprechend gehören die Sonographie von Abdomen und Pleura sowie die Erhebung eines Gefäßstatus zur Basisdiagnostik.

\section{Bildgebende Diagnostik}

Sie schließt sich in Form von Röntgen, CT und ggf. MRT an und ist notwendig, um stabile von instabilen Verletzungen unterscheiden und eine exakte Klassifikation der jeweiligen Läsionen vornehmen zu können. Eine Verletzung ist dann als instabil anzusehen, wenn eine Gefahr für eine neurologische Beeinträchtigung durch Verlegung des Spinalkanals besteht oder wenn gravierende unphysiologische Fehlstellungen des Achsorgans existieren bzw. durch Mobilisation eintreten können und somit Fehlbelastungen primär nicht betroffener Wirbelsäulensegmente oder von Gelenken der unteren Extremitäten die Folge wären. Bezogen auf die A-B-C-Klassifikation nach Magerl et al. [17] sind alle Verletzungen einschließlich und jenseits des Kneifzangenbruchs (Typ A2.3) als instabil einzustufen. 

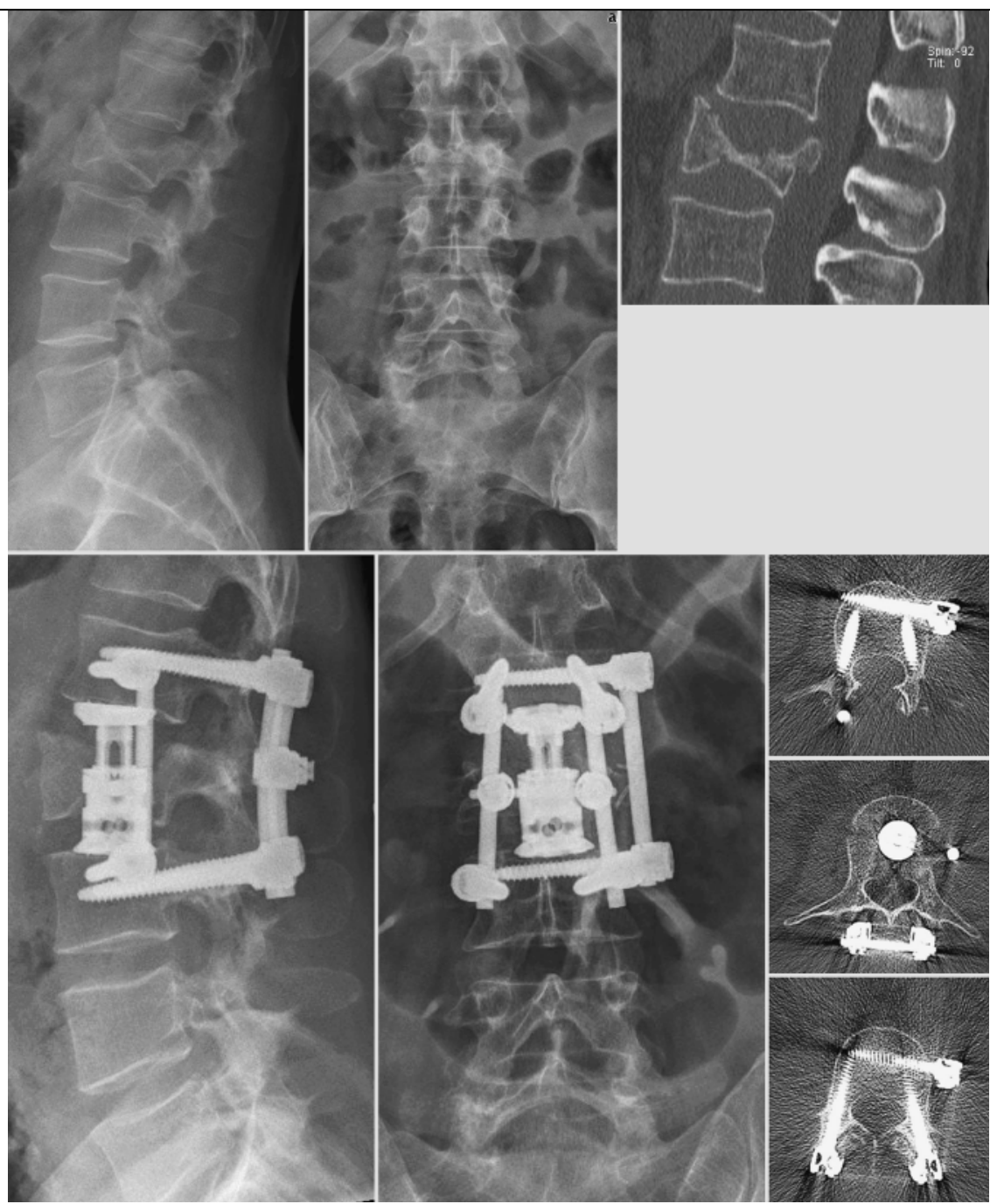

Abb. 3 \ 53-jähriger Patient mit Kneifzangenberstungsbruch (Typ A3.3.1) nach Verkehrsunfall, zusätzlich zu Fixateur interne und ventralem Cage Durchführung einer ventralen Einstabinstrumentation, um Einsinken des Cages zu vermeiden

Röntgen. Bei der Beurteilung der initialen Röntgenaufnahmen von BWS und LWS ist im lateralen Bild sowohl auf den Verlust der Wirbelkörperhöhe und auf die Kyphosewinkel des frakturierten Wirbels selbst als auch auf den mono- und bisegmentalen Grunddeckplattenwinkel unter Einschluss der angrenzenden Wirbelkörper zu achten. Zeigt der verletzte Wirbel im Gegensatz zu reinen Kompressionsfrakturen (Typ A) eine Kyphose von mehr als $20^{\circ}$ oder ist ein deutliches Auseinanderweichen der Dornfortsätze im Vergleich zu kranial und kaudal benachbarten Segmenten nachweisbar, ist das Vorliegen einer Flexions-Distraktions-Verletzung (Typ B) wahrscheinlich. Die Divergenz der Dornfortsätze findet sich dann häufig im a.-p. Strahlengang wieder. Ein sprunghaftes Abweichen der Dornfortsätze aus der Mittellinie, eine Asymmetrie der als "Augen“ sichtbaren Bogenwurzeln sowie Querfortsatz- und Rippenköpfchenfrakturen deuten auf eine Rotationsverletzung (Typ C) hin.

Computertomographie. Durch sie ist eine bessere Einschätzung der Fraktur möglich. Sie ist heutzutage als Standard zur Klassifikation einer Wirbelfraktur zu fordern. Axiale Schichtbilder und multiplanare Rekonstruktionen erlauben z. B. Berstungsspaltbrüche von inkompletten Berstungsbrüchen zu unterscheiden und sind wegweisend für eine konsequente klassifikationsorientierte Therapie. Zudem lassen sich Einengungen des Spinalkanals besser beurteilen und die Konfiguration von Hinterkantenfragmenten
Zusammenfassung $\cdot$ Abstract

Trauma Berufskrankh 2007 · 9:149-156

DOI 10.1007/s10039-006-1150-y

(c) Springer Medizin Verlag 2006

\section{S. Katscher $\cdot$ C. Josten}

\section{Wirbelsäulenverletzungen des thorakolumbalen Übergangs. Versorgungsstrategien}

\section{Zusammenfassung}

Eine einheitliche Behandlungsstrategie thorakolumbaler Wirbelfrakturen hat sich lange Zeit nicht etablieren können. Heute ist bei stabilen Frakturen mit intakter Hinterkante (A1.2) und ohne wesentliche Kyphosierung die frühfunktionelle Therapie allgemein akzeptiert. Bei instabilen Frakturen werden jedoch noch immer verschiedene Konzepte propagiert. Dies betrifft v. a. die Indikation und Form der Durchführung einer additiven ventralen Stabilisierung nach Reposition und dorsaler Instrumentation bei den verschiedenen Formen der Berstungsfrakturen vom Typ A-C. In der vorliegenden Arbeit soll ein differenziertes Behandlungsregime von Wirbelfrakturen des thorakolumbalen Übergangs in Abhängigkeit von der Klassifikation der Verletzung, der neurologischen Begleitsymptomatik und dem Lebensalter der Patienten vorgestellt werden.

\section{Schlüsselwörter}

Thorakolumbale Wirbelfraktur - Endoskopie - Navigation - Expandierbarer Cage · Knochenspan

\section{Injuries to the thoracolumbar spine. Treatment strategies}

\section{Abstract}

For a long time there was no uniform treatment strategy for fractures of the thoracolumbar spine. Early functional treatment is now generally accepted as course for stable fractures with intact posterior wall (A1.2) in the absence of substantial kyphosis. In case of unstable burst fractures, however, there are still various competing treatment strategies. This applies especially in the case of the indications for and the type of additive ventral stabilization in the various forms of type A-C burst fractures. This paper presents treatment options for thoracolumbar vertebral fractures in dependency of the classification of the injury, the concomitant neurological symptoms and the age of the patient.

\section{Keywords}

Thoracolumbar fracture - Endoscopy - Navigation - Expandable cage $\cdot$ Bone graft 


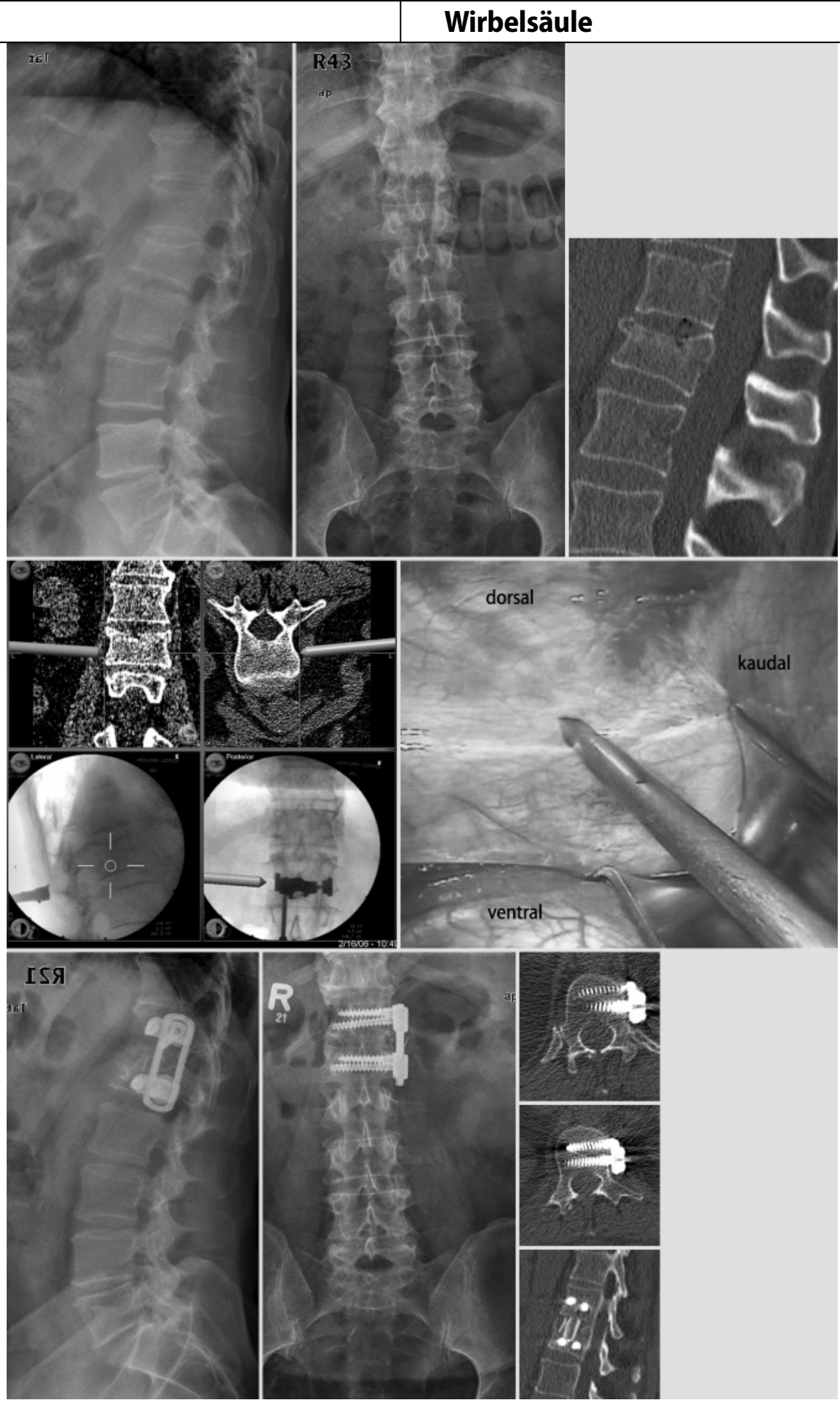

Abb. $4 \Delta$ Endoskopisch und navigationsgestützt assistierte ventrale monosegmentale Spondylodese bei 56-jährigem Patienten mit kranialem Berstungsbruch von LWK1 (Typ A3.1)

exakt darstellen, was wiederum einen prädiktiven Wert für die Wahrscheinlichkeit einer Reposition der Fragmente durch Lagerung und Längszug sowie die dorsale Instrumentierung besitzt.

MRT. Es ist zur Erhebung der Klassifikation und Festlegung der weiteren Therapie bisher nicht regelhaft gefordert. An- diesem Grund führen wir bei mehr als $15^{\circ}$ kyphosierten scheinbaren Keil- (Typ A1.2) und inkompletten Berstungsfrakturen (Typ A3.1) eine Magnetresonanztomographie durch, um das Therapieregime festzulegen. Weiterhin ist bei frischen posttraumatischen neurologischen Ausfällen ohne feststellbare Verletzungen im Nativröntgen oder CT eine MRT-Untersuchung notwendig, um zwar seltene, aber mögliche unfallbedingte intraspinale oder epidurale Hämatome nachzuweisen.

\section{Behandlung}

Ihre Ziele bei thorakolumbalen Wirbelfrakturen sind

- die Reduktion bzw. Vermeidung neurologischer Ausfälle,

- die Reposition von Deformierungen,

- die Wiederherstellung der Stabilität und

- die Vermeidung bandscheibenbedingter Folgeschäden.

\section{Frühfunktionelle Therapie stabiler Frakturen}

Die konservative Therapie stellt für die stabilen $\mathrm{A}_{1} / \mathrm{A}_{2}$-Frakturen die adäquate Option dar. Dies beinhaltet nach Rückgang der Akutschmerzen durch Analgetikaapplikation eine Mobilisation ab dem 1. posttraumatischen Tag, begleitet von einer stabilisierenden krankengymnastischen und physikalischen Behandlung. Dabei kann in der Regel auf weitere additive Maßnahmen, wie Orthesen und Korsettanlage, verzichtet werden. Aufgrund der bekannten Problematik der Mitbeteiligung von dorsalen Bandverletzungen empfiehlt sich nach 3-4 Tagen eine Röntgenaufnahme im Stehen. Sollte es hier zu keiner weiteren Kyphosierung gekommen sein, kann die funktionelle Behandlung fortgesetzt werden. Obsolet sind langwöchige Ruhigstellungen im Gipskorsett bzw. im Bett.

Trotz der stabilen Fraktur wird im Behandlungsverlauf bei konservativer Therapie fast regelhaft eine sekundäre Kyphosierung (im Durchschnitt 3-5 $5^{\circ}$ beobachtet. Aus diesem Grund ist im seltenen Fall einer A1.2-Fraktur mit initialer Kyphose von $15^{\circ}$ und mehr bei MR-morphologisch sicher intaktem dorsalem Ligamentkom- 
plex eine isoliert ventrale monosegmentale Fusion beim jungen Menschen diskussionswürdig, da eine Ausheilung mit $20^{\circ}$ Kyphose neben der Degeneration der Bandscheibe mit einer relativen Instabilität verbunden ist und zu einer Fehlbelastung der Facettengelenke und somit einer vorzeitigen Spondylarthrose mit chronischen Beschwerden führen kann.

\section{Operative Therapie instabiler Verletzungen}

Die instabile Wirbelfraktur sollte frühestmöglich operativ reponiert und stabilisiert werden, da kurz nach dem Trauma die Wahrscheinlichkeit einer optimalen Reposition am höchsten ist. Eine lordosierende Lagerung mit guter Unterpolsterung von Thorax und Becken ist hierbei hilfreich und verbessert gleichzeitig durch Vermeidung einer Kompression der Vena cava den Blutrückstrom zum Herzen (- Abb. 1).

Praktisch führen wir - außer beim polytraumatisierten Patienten - bei den 8o90\% aller instabilen Frakturen des thorakolumbalen Übergangs repräsentierenden Berstungsspalt- und kompletten Berstungsbrüchen vom Typ A-C sowie inkompletten Berstungsbrüchen vom Typ B/C die dorsale Reposition und Instrumentierung, wenn logistisch und patientenbedingt möglich, am Aufnahmetag durch. Dabei sollten möglichst lange und kräftige, sich in den Pedikeln verklemmende Schrauben verwendet werden, was vorher am CT entsprechend ausgemessen werden kann. Außerdem empfiehlt es sich, die Facettengelenke der Segmente, die definitiv versteift werden sollen, mit dem Luer aufzubrechen, um eine sekundäre Verknöcherung und Spondylodese anzuregen. Eine zusätzliche Spongiosaanlagerung wird kontrovers diskutiert und von uns nicht durchgeführt.

Nicht nur die Wiederherstellung des lokalen Alignements und der knöchernen Struktur des Wirbelkörpers, sondern auch ganz wesentlich die Integrität der Bandscheibe bestimmen dauerhafte Stabilität und Funktionalität der Wirbelsäule. Bei Berstungsfrakturen werden die angrenzenden Bandscheiben, insbesondere der Nucleus pulposus, durch direkten Maximaldruck im Moment des Traumas und
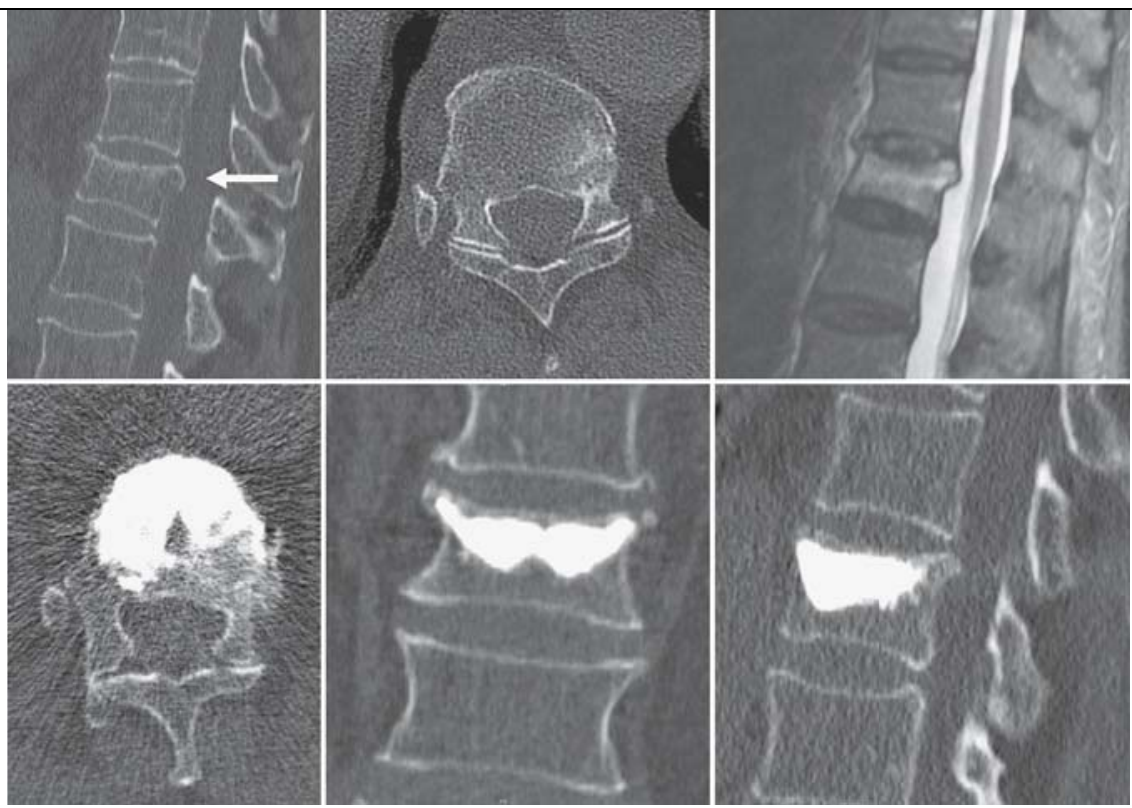

Abb. $5 \Delta$ 85-jähriger Patient mit kranialem Berstungsbruch bei Osteoporose (T-Score=-3,1), Aufrichtung der Fraktur und partielle Reposition des Hinterkantenfragments durch Kyphoplastie
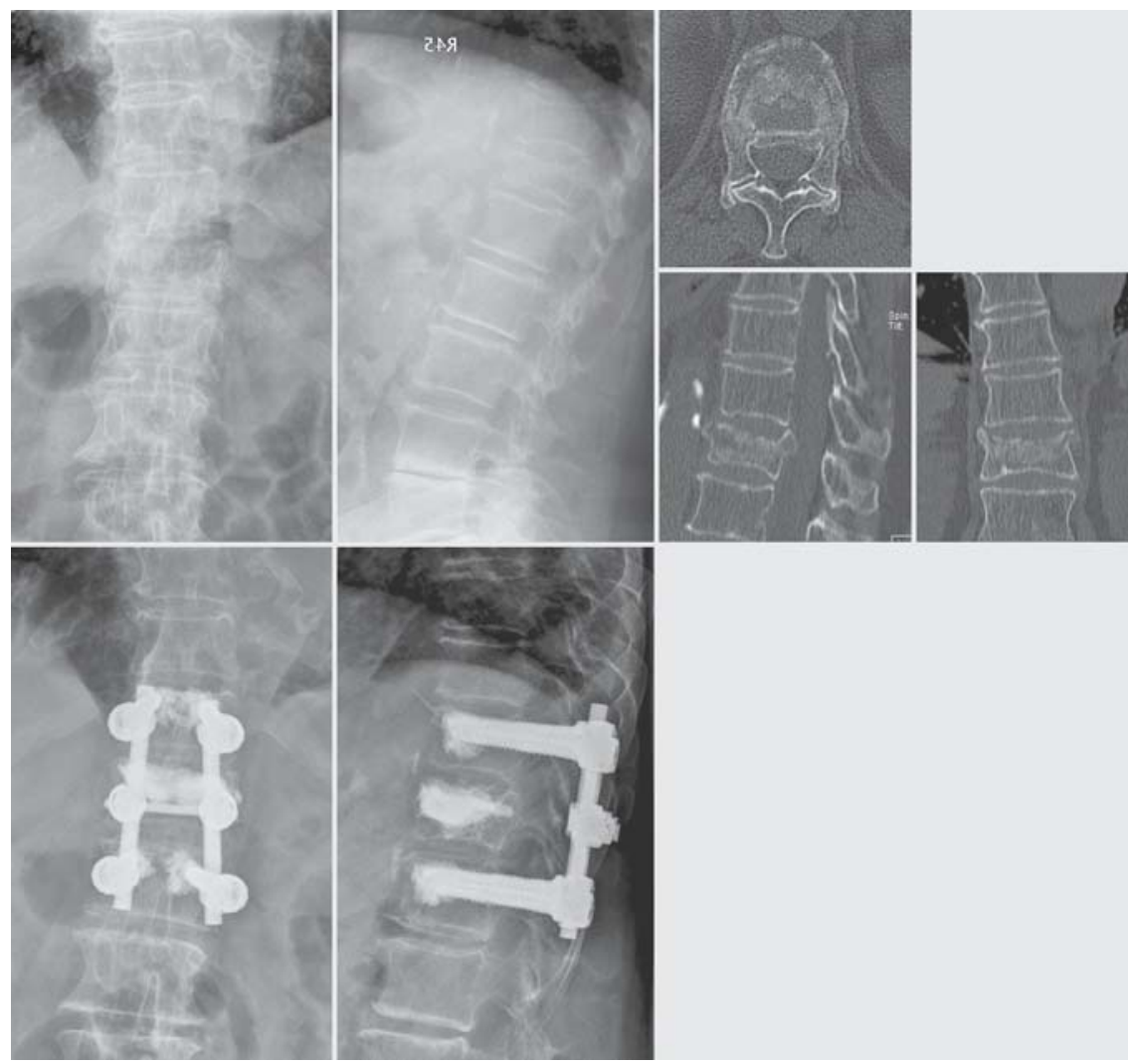

Abb. $6 \triangle$ 78-jährige Patientin mit komplettem Berstungsbruch (Typ A3.3) von BWK12 bei Osteoporose (T-Score $=-2,2)$, Instrumentierung und Stabilisierung durch zementaugmentierten Fixateur interne und Kyphoplastie, aus Katscher et al. [14]

durch die scharfkantigen Fragmente des berstenden Wirbelkörpers zerstört und ihrerseits in den frakturierten Wirbel imprimiert. Dadurch kommt es zu einer „Weich- teilschädigung “ der Wirbelsäule, die als irreversibel anzusehen ist und letztendlich das Schicksal für den weiteren Heilungsverlauf bestimmt. Auch bei Berstungs- 


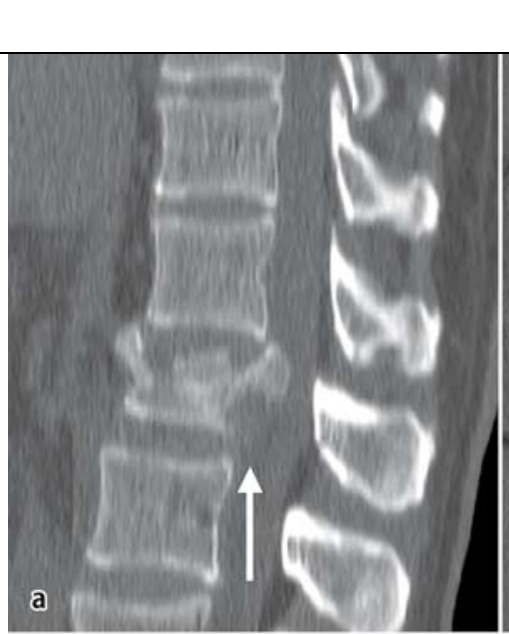

Wirbelsäule
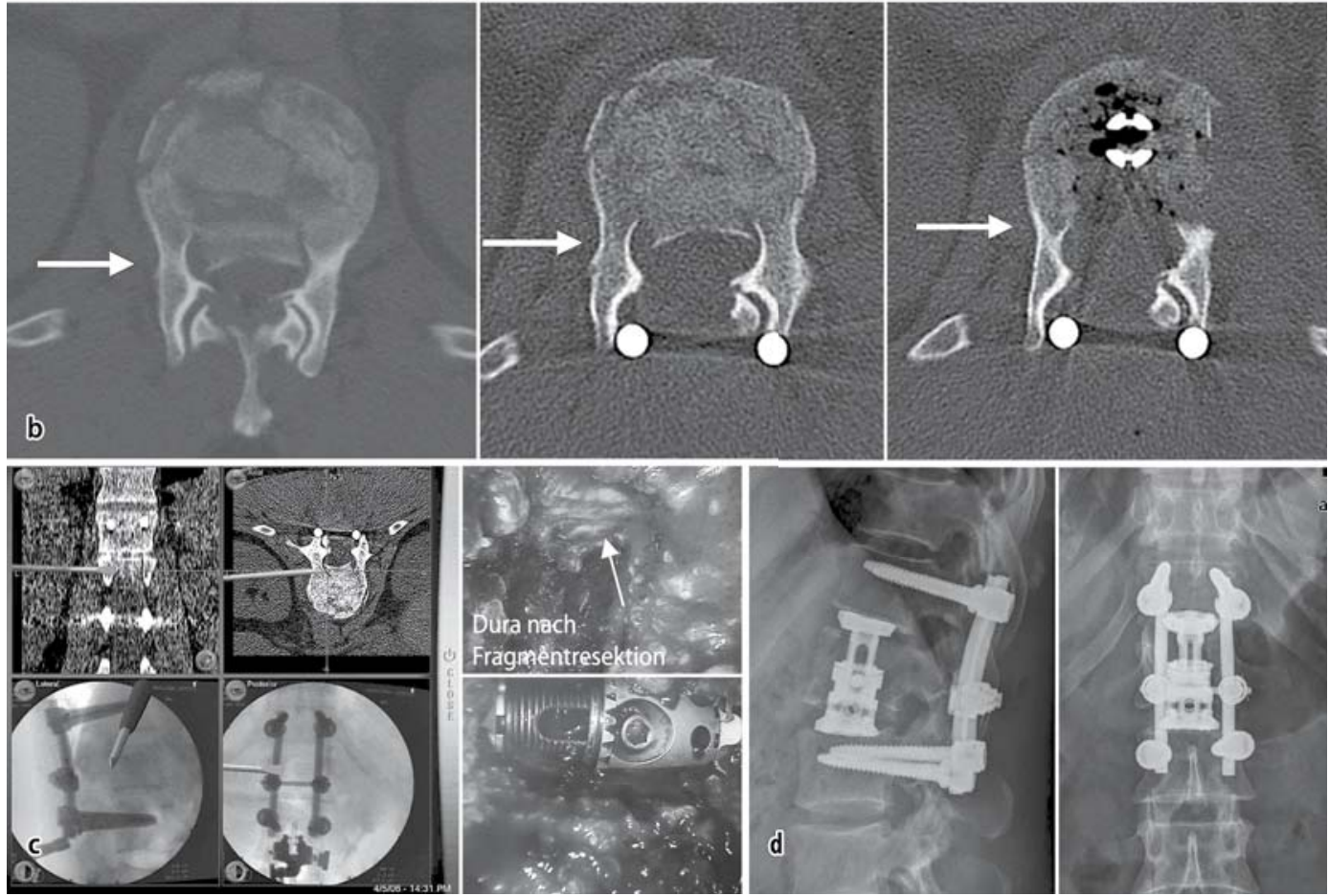

Abb. 74 39-jähriger Patient mit Konus-Kauda-Syndrom durch großes Hinterkantenfragment (Pfeil) bei instabiler C1.3-Fraktur von LWK1 nach Gerüststurz, a-f CT, a, d Unfall, b, e nach dorsaler Instrumentierung mit Laminektomie, $\mathbf{c}, \mathbf{f}$ nach ventraler Spinalkanalclearance, $\mathbf{g}$ thorakoskopisch assistierte, navigationsgestützte Hinterkantenresektion, Cageinterposition, $\mathbf{h}$ Röntgenkontrolle nach Mobilisation

spaltbrüchen muss man davon ausgehen, dass beide benachbarten Bandscheiben geschädigt sind, was CT-morphologisch oft erst nach einigen Wochen als Vakuumphänomen im Zwischenwirbelraum imponiert (• Abb. 2). Diese Bandscheibenschädigungen sind als Ursache für die erheblichen sekundären Korrekturverluste nach alleiniger dorsaler Instrumentierung anzusehen $[9,10,13,15,19]$. Zusätzlich zu diesen klinischen Erfahrungen zur bedeutenden Pathologie des ventralen "Druckpuffers" und „Querträgers“ Bandscheibe hat die Einführung minimalinvasiver, rein endoskopischer oder endoskopisch assistierter „mini open“-Techniken $[1,5,6,11,21] \mathrm{zu}$ einer deutlichen Zunahme der ventralen Verfahren geführt.
Nur die konsequente Ausräumung der zerstörten Bandscheiben mit einem entsprechenden interkorporalen Ersatz und ggf. einer additiven Instrumentierung können den sekundären Korrekturverlust vermeiden. Dabei ist die Frage, ob kortikospongiöser Knochenspan oder Cage mit oder ohne Stabilisierung zu besten Ergebnissen führen, noch nicht endgültig geklärt und somit Zielstellung verschiedener Studien. Dem Vorteil der Osteointegration des autogenen Spans stehen die Gefahr einer Volumenabnahme desselben und einer mangelhaften Einheilung, welche mit $10-30 \%$ angegeben wird [4, $18,20]$, sowie die individuell sehr unterschiedliche Größe des gewonnenen Spans und die Entnahmemorbidität gegenüber. Aus diesem Grund wird bei Berstungs- spalt- und kompletten Berstungsbrüchen (Typ A-C) zusätzlich zur dorsalen Instrumentierung ventral in den meisten Zentren ein expandierbarer Cage mit möglichst hohem Grundplattenquerschnitt bisegmental eingebracht. Im Gegensatz zu jungen Patienten mit guter Knochenqualität haben wir bei älteren Patienten mit ventraler „stand alone“-Cage-Interposition in einigen Fällen ein sekundäres Einsinken der Cages beobachten müssen. Deshalb führen wir mittlerweile bei Patienten oberhalb des 50 . Lebensjahrs eine zusätzliche ventrale Instrumentation mit einem Einstabsystem durch (• Abb. 3). Alternativ wird ventral zusätzlich zum Cage auch eine winkelstabile Instrumentation empfohlen. Sowohl bei der Bandscheiben- und partiellen Wirbelresekti- 
on als auch bei Platzierung der ventralen Schrauben hat sich bei endoskopisch assistierter Operationstechnik der Einsatz der Navigation bewährt und verkürzt zusätzlich die intraoperative Durchleuchtungsdauer. Eine Materialentfernung ist bei kombiniert dorsoventral bisegmentalem Vorgehen und komplikationslosem Verlauf aufgrund des geschlossenen Kraftkreises nicht erforderlich.

Liegt ein inkompletter Berstungsbruch vom Typ B/C vor, können zusätzlich zur dorsalen Instrumentierung ventral ein monosegmentaler Span mit Instrumentation oder ein Cage mit lateral angelagerter, aus den Wirbelkörpertrümmern gewonnener Spongiosa eingebracht werden. Die dorsale Materialentfernung erfolgt nach 6-9 Monaten

Inkomplette Berstungsbrüche vom Typ A (A3.1) können von der Verletzungsmorphologie und Biomechanik prinzipiell nur von ventral stabilisiert werden. Dorsale ligamentäre Läsionen müssen in diesen Fällen - wie oben erwähnt - jedoch sicher ausgeschlossen werden. Nach Ausräumung der Bandscheibe werden ein Knochenspan oder ein kleiner Cage interponiert und die Reposition mit einem winkelstabilen Implantat gesichert (- Abb. 4). Falls eine ausreichende Reposition durch Lagerung und lokalen Druck auf die Dornfortsätze nicht erreicht wird, können von dorsal perkutan eingebrachte Schanz-Schrauben im Sinne eines temporären Fixateur externe zur Reposition verwendet und nach Fixierung des ventralen Implantats wieder entfernt werden.

\section{Sonderfälle}

Die alleinige dorsale Instrumentierung ist aufgrund der erwähnten sekundären Korrekturverluste nur wenigen Fällen vorbehalten. Dazu zählen neben den seltenen instabilen kindlichen Wirbelfrakturen beim Erwachsenen die horizontalen diskoligamentären oder ossären B-Verletzungen ohne Trümmerzone im Bereich des Wirbelkörpers.

Eine spezielle Konstellation liegt auch bei den osteoporotischen Frakturen vor. Vom eigentlichen Klassifikationssystem her stabile Brüche ohne Hinterkantenbeteiligung werden aufgrund des „Hohlraums“ zwischen den Grund- und Deck- platten zu instabilen Läsionen und können unter Mobilisation in einer Vertebra plana enden. Für diese Verletzungen stellen die Vertebroplastie sowie die mit geringerer Komplikationsrate assoziierte Kyphoplastie sehr gute therapeutische Optionen dar. Bei frischen osteoporotischen inkompletten Berstungsbrüchen ohne großes Hinterkantenfragment oder bei älteren, schmerzhaften, in Konsolidierung befindlichen und im MRT noch aktiven Berstungsbrüchen kann die Kyphoplastie ebenfalls vorsichtig mit weit ventral platziertem Ballon angewendet werden [3] (- Abb. 5). Bei frischen Berstungsspalt- oder Berstungsfrakturen dagegen sollte eine dorsale Stabilisierung ohne wesentliche Reposition erfolgen. Um Implantatausbrüche zu vermeiden, sind neben kräftigen Schrauben eine längerstreckige Instrumentation oder Zementaugmentierung des Fixateurs zu empfehlen. Eine Kombination mit einer sparsamen Kyphoplastie des frakturierten Wirbels ist hierbei möglich (• Abb. 6). Eine Kombination aus Kyphoplastie und nicht zementiertem Fixateur können wir dagegen aus eigener Erfahrung nicht empfehlen.

Bei instabilen Frakturen mit neurologischen Ausfällen ist die schnellstmögliche Reposition Ziel aller primären Maßnahmen. Die Standardtherapie ist hierbei zunächst die dorsale Instrumentierung. Weitere Maßnahmen zur Dekompression bei einer persistierenden Kompression sind die kontrovers diskutierte, partielle oder vollständige Laminektomie und der Versuch des direkten Zurückstößelns oder Bergens eines Hinterkantenfragments von dorsal. Beim kombinierten Eingriff ist auch eine Dekompression über den ventralen Zugang möglich. Ansonsten sollten bei postoperativ ausbleibender oder unvollständiger Remission der neurologischen Ausfälle die additive ventrale Dekompression und Stabilisierung möglichst rasch durchgeführt werden. Die endoskopische Technik ermöglicht bei kalkulierbarem Risiko eine gute Spinalkanalclearance [1] ( $\bullet$ Abb. 7). Bei neurologisch intakten Verhältnissen jedoch ist eine Hinterkantenresektion wegen möglicher Verletzung epiduraler Venen oder Duraläsion nicht zu empfehlen. Außerdem ist sie in diesen Fällen auch nicht nötig, da im weiteren Verlauf oft eine spontane Erwei- terung des Spinalkanals im Rahmen des so genannten „Remodellings“ beobachtet wird $[7,12,16,22]$.

\section{Fazit}

Stabile Frakturen des thorakolumbalen Übergangs mit intakter Hinterkante (A1.2) und ohne wesentliche Kyphosierung $\left(<15^{\circ}\right)$ werden heutzutage frühfunktionell behandelt. Instabile Läsionen dagegen erfordern eine operative Therapie, welche bis auf wenige Ausnahmen aufgrund der speziellen Pathologie des Wirbel-Bandscheiben-Konstrukts durch eine dorsoventrale Instrumentierung unter fakultativem Einsatz endoskopischer und navigationsgestützter Techniken realisiert wird.

Offen sind die Fragen nach dem dauerhaft sichersten ventralen Spacer: Knochenspan vs. Cage oder perspektivischem Einsatz von Knochenersatzstoffen oder einer "Frakturbandscheibe". Weiterhin bleibt abzuwarten, ob radiologisch erfassbare geringere Korrekturverluste langfristig zu besseren klinischen Ergebnissen führen und somit kostenintensive operative Rekonstruktionen rechtfertigen.

\section{Korrespondierender Autor}

\section{Dr. S. Katscher}

Chirurgische Klinik und Poliklinik III, Universität Leipzig

Liebigstraße 20a, 04103 Leipzig

Sebastian.Katscher@medizin.uni-leipzig.de

Interessenkonflikt. Es besteht kein Interessenkonflikt. Der korrespondierende Autor versichert, dass keine Verbindungen mit einer Firma, deren Produkt in dem Artikel genannt ist, oder einer Firma, die ein Konkurrenzprodukt vertreibt, bestehen. Die Präsentation des Themas ist unabhängig und die Darstellung der Inhalte produktneutral.

\section{Literatur}

1. Beisse R, Mückley T, Schmidt MH et al. (2005) Surgical technique and results of endoscopic anterior spinal canal decompression. J Neurosurg Spine 2: 128-136

2. Blattert TR, Glasmacher S, Josten C (2006) Ballonkyphoplastie: Indikation, Diagnostik, operative Technik, Ergebnisse (Teil 2: Ergebnisse). Aktuelle Traumatol 36: 14-17

3. Boszczyk BM, Bierschneider M, Potulski M (2002) Erweitertes Anwendungsspektrum der Kyphoplastie zur Stabilisierung der osteoporotischen Wirbelfraktur. Unfallchirurg 105: 952-957 
4. Briem D, Rueger JM, Linhart W (2003) Einheilung autogener Transplantate nach dorsoventraler Instrumentierung instabiler Frakturen der thorakolumbalen Wirbelsäule. Unfallchirurg 106: 195-203

5. Bühren V (2003) Verletzungen der Brust- und Lendenwirbelsäule. Unfallchirurg 106: 55-69

6. Bühren V, Beisse R, Potulski M (1997) Minimal-invasive ventrale Spondylodesen bei Verletzungen der Brust- und Lendenwirbelsäule. Chirurg 68: 10761084

7. Dai LY (2001) Remodelling of the spinal canal after thoracolumbar burst fractures. Clin Orthop 2001: $119-123$

8. Daniaux H (1986) Transpedikuläre Reposition und Spongiosaplastik bei Wirbelkörperbrüchen der unteren Brust- und Lendenwirbelsäule. Unfallchirurg 89: 197-213

9. Eysel P, Rompe JD, Hopf C et al. (1994) Die Bedeutung der Bandscheibe für den Repositionsverlust operativ stabilisierter Frakturen der Rumpfwirbelsäule. Unfallchirurg 97: 451-457

10. Feil J, Wörsdorfer O (1992) Ventrale Stabilisierung im Bereich der Brust- und Lendenwirbelsäule. Chirurg 63: 856-865

11. Josten C, Katscher S, Gonschorek O (2005) Therapiekonzepte bei Frakturen des thorakolumbalen Überganges und der Lendenwirbelsäule. Orthopäde 34: 1021-1034

12. Karlsson MK, Hasserius R, Sundgren $P$ et al. (1997) Remodelling of the spinal canal deformed by trauma. J Spinal Disord 10: 157-161

13. Katscher S, Verheyden $P$, Gonschorek $O$ et al (2003) Thorakolumbale Wirbelfrakturen nach konservativer und operativer Behandlung - Abhängigkeit des Korrekturverlustes von der Höhenlokalisation. Unfallchirurg 106: 20-27

14. Katscher S, Blattert T, Glasmacher $S$ et al. (2006) Fehler und Komplikationen bei der Kyphoplastie. Aktuelle Traumatol 36: 23-28

15. Knop C, Blauth M, Bühren V et al. (2000) Operative Behandlung von Verletzungen des thorakolumbalen Übergangs. Teil 2: Operation und röntgenologische Befunde. Unfallchirurg 103: 1032-1047

16. Kuner EH, Schlickewei W, Hauser U et al. (1996) Zur Wiederherstellung der lichten Weite des Spinalkanals durch Fixateur-interne-Instrumentation und Remodelling. Chirurg 67: 531-538

17. Magerl F, Aebi M, Gertzbein SD et al. (1994) A comprehensive classification of thoracic and lumbar injuries. Eur Spine J 3: 184-201

18. Moll R, Schindler G, Weckbach A (2002) Beurteilung der ventralen Stabilisierungstechniken thorakolumbaler Wirbelsäulenverletzungen mit Hilfe der Computertomographie. Fortschr Roentgenstr 174: 880-886

19. Resch H, Rabl M, Klampfer H et al. (2000) Operative vs. konservative Behandlung von Frakturen des thorakolumbalen Übergangs. Unfallchirurg 103: 281-288

20. Schultheiss M, Sarkar M, Arand M et al. (2005) Solvent-preserved, bovine cancellous bone blocks used for reconstruction of thoracolumbar fractures in minimally invasive spinale surgery - first clinical results. Eur Spine J 14/2: 192-196

21. Verheyden P, Hoelzl A, Lill H et al. (2004) The endoscopically assisted simultaneous posteroanterior reconstruction of the thoracolumbar spine in prone position. Spine J 4: 540-549

22. Wessberg $P$, Wang $Y$, Irstam $L$ et al. (2001) The effect of surgery and remodelling on spinal canal measurements after thoracolumbar burst fractures. Eur Spine J 10: 55-63

23. Woltmann A, Bühren V (2004) Schockraummanagement bei Verletzungen der Wirbelsäule im Rahmen eines Polytraumas. Unfallchirurg 107: 911-918 